This is a non-final version of an article published in final form in:

Jenkinson C, Elliott V, Menon U, Apostolidou S, Fourkala OE, Gentry-Maharaj A, Pereira SP, Jacobs I, Cox TF, Greenhalf W, Timms JF, Sutton R, Neoptolemos JP, Costello E. Evaluation in prediagnosis samples discounts ICAM-1 and TIMP-1 as biomarkers for earlier diagnosis of pancreatic cancer. Journal of proteomics. 2015 Jan 15;113:400-2. 10.1016/j.jprot.2014.10.001. Downloaded from UCL Discovery: http://discovery.ucl.ac.uk/1451917

\title{
ARTICLE
}

\section{Evaluation in pre-diagnosis samples discounts ICAM-1 and TIMP-1 as biomarkers for earlier diagnosis of pancreatic cancer}

\author{
C. Jenkinson ${ }^{1,2}$, V. Elliott ${ }^{1}$, U. Menon ${ }^{3}$, S. Apostolidou ${ }^{3}$, O.E. Fourkala ${ }^{3}$, A. Gentry-Maharaj $^{3}$,
} S.P. Pereira, ${ }^{4,5}$, I. Jacobs ${ }^{3,5}$ T.F. Cox ${ }^{1}$, W. Greenhalf', J.F. Timms ${ }^{3}$, R. Sutton ${ }^{1,2}$, J.P. Neoptolemos ${ }^{1,2}$ and E. Costello $0^{1,2}$.

${ }^{1}$ NIHR Liverpool Pancreas Biomedical Research Centre, ${ }^{2}$ Department of Molecular and Clinical Cancer Medicine, University of Liverpool, Daulby Street, Liverpool, L69 3GA, UK; ${ }^{3}$ Women's Cancer, Institute for Women's Health, University College London, Gower Street, London, WC1E 6BT, UK; ${ }^{4}$ Institute for Liver and Digestive Health, University College London, Upper 3rd Floor Royal Free Hospital, Pond Street, London NW3 2QG, UK; ${ }^{5}$ Department of Gastroenterology, University College Hospitals NHS Foundation Trust, 235 Euston Road, London, NW1 2BU, UK; ${ }^{5}$ Faculty of Medical and Human Sciences, 1.018 Core Technology Facility, University of Manchester, Grafton Street, M13 9NT, UK

To whom correspondence should be addressed:

Eithne Costello, NIHR Liverpool Pancreas Biomedical Research Unit, University of Liverpool, Daulby Street, Liverpool L69 3GA, UK. Email: ecostell@liverpool.ac.uk; Telephone +441517064178; FAX: +441517065826

\begin{abstract}
Circulating intercellular adhesion molecule-1 (ICAM-1) and tissue inhibitor of metalloproteinases-1 (TIMP-1) have been widely proposed as potential diagnostic biomarkers for pancreatic ductal adenocarcinoma (PDAC). We report on serum protein levels prior to clinical presentation of pancreatic cancer. Serum ICAM-1 and TIMP-1 were measured by ELISA in two case-control sets 1) samples from patients diagnosed with pancreatic cancer $(n=40)$, chronic pancreatitis $(n=20)$, benign jaundice due to gall stones $(n=20)$ and healthy subjects $(n=20) ; 2)$ a preclinical set from the UK Collaborative Trial of Ovarian Cancer Screening biobank of samples collected from 27 post-menopausal women 0-12 months prior to diagnosis of pancreatic cancer and controls matched for date of donation and centre. Levels of ICAM-1 and TIMP-1 were significantly elevated in set 1 in PDAC patients with jaundice compared to PDAC patients without jaundice and both proteins were elevated in patients with jaundice due to gall stones. Neither protein was elevated in samples taken 0-12 months prior to PDAC diagnosis compared to non-cancer control samples. In conclusion, evaluation in pre-diagnosis samples discounts ICAM-1 and TIMP-1 as biomarkers for earlier diagnosis of pancreatic cancer. Failure to account for obstructive jaundice may have contributed to the previous promise of these candidate biomarkers.
\end{abstract}


Key words: ICAM-1, TIMP-1, pancreatic cancer, serum biomarker, UKCTOCS

\section{To the editor}

Pancreatic ductal adenocarcinoma (PDAC) is usually diagnosed when the disease is advanced. This limits therapeutic options and biomarkers for earlier diagnosis are sought (1). Using blood taken at diagnosis of PDAC to identify biomarkers poses intrinsic challenges. The levels of blood proteins at diagnosis may accurately represent advanced disease, but might not necessarily reflect levels prior to diagnosis. In addition, the sensitivity of some markers for the detection of PDAC over healthy controls was recently shown to be considerably higher for PDAC patients in the presence of obstructive jaundice compared to PDAC patients in the absence of obstructive jaundice (2). Two serum proteins, identified in several studies as potential diagnostic biomarkers due to their upregulation in PDAC, are ICAM-1 (3-6) and TIMP-1 (3-5, 7-10). In order to determine whether ICAM-1 and TIMP-1 levels are affected by biliary obstruction we compared samples (Liverpool cohort) taken at diagnosis from patients with PDAC (in the presence or absence of jaundice), chronic pancreatitis, benign biliary obstruction or from healthy controls. In patients with PDAC, both ICAM-1 and TIMP-1 levels were significantly higher than in healthy controls (Figure 1a and b), as previously reported (3-10). PDAC patients with biliary obstruction (bilirubin levels $>20$ $\mu \mathrm{mol} / \mathrm{L})$ had significantly higher levels of both ICAM-1 $(p<0.001)$ and TIMP-1 $(p=0.024)$ than PDAC patients without biliary obstruction (bilirubin levels $<20 \mu \mathrm{mol} / \mathrm{L}$ ). ICAM-1 and TIMP-1 levels were also elevated in patients with biliary obstruction due to gall stones $(p<0.0001$ for ICAM-1 and $p=0.0001$ TIMP-1). Finally these proteins only distinguished PDAC from chronic pancreatitis when the PDAC patients had biliary obstruction $(p=0.004$ for ICAM- 1 and $\mathrm{p}=0.014$ for TIMP-1, Figure 1 $\mathrm{a}$ and $\mathrm{b}$ ).

Secondly, to determine if these proteins are raised prior to clinical presentation of pancreatic cancer, we analysed pre-diagnostic samples collected as part of the UK Collaborative Trial of Ovarian Cancer Screening (UKCTOCS)(11), from individuals who went on to develop pancreatic cancer during the study, and compared them with their time matched cancer-free controls. No significant differences were observed between serum ICAM-1 or TIMP-1 from UKCTOCS cases $(n=27)$ and matched controls $(n=27)$ from the time period $0-12$ months prior to PDAC diagnosis (Figure $1 \mathrm{c}$ and $\mathrm{d}$ ). Of note, although all of the individuals in the UKCTOCS cohort were female, the Liverpool cohort contained both males (59/100) and females (41/100). Neither ICAM-1 nor TIMP-1 were associated with gender or age in any group.

Our study shows that although the levels of both ICAM-1 and TIMP-1 were significantly raised at the time of diagnosis in cancer patients compared to healthy controls, the absolute increases observed in patients in the absence of biliary obstruction were relatively small compared to those seen in the presence of obstruction. Moreover, neither marker was effective in distinguishing patients with chronic pancreatitis from PDAC patients in the absence of biliary obstruction. We conclude that the initial promise of these two candidates as markers of pancreatic cancer was artificially inflated due to failure to account for biliary obstruction in cancer patients. Secondly we show that the levels of ICAM-1 and TIMP-1 are not significantly elevated in the months leading to a diagnosis of pancreatic cancer, and provide little support for the notion that either of these markers will be effective for early detection of pancreatic cancer. 


\section{Acknowledgements}

This work was supported by The National Institute for Health Research Liverpool Pancreas Biomedical Research Unit, North West Cancer Research grant CR976 and Cancer Research UK grant A12790. UKCTOCS was core-funded by the Medical Research Council, Cancer Research UK, and the Department of Health with additional support from the Eve Appeal, Special Trustees of Bart's and the London, and Special Trustees of UCLH. UKCTOCS researchers were supported by the National Institute for Health Research University College London Hospitals Biomedical Research Centre.

\section{Figure legend}

Figure 1. Analysis of ICAM-1 and TIMP-1 in serum samples. Box and whisker plots for serum levels of ICAM-1 (Human sICAM-1/CD54 Quantikine ELISA Kit, R\&D Systems, Minneapolis, USA) and TIMP-1 (Human TIMP-1 Quantikine ELISA Kit, R\&D Systems, Minneapolis, USA) measured in the Liverpool cohort samples (a) \& (b) and UKCTOCS samples (c) \& (d). Liverpool cohort details - Gender M/F, Median Age in years (IQR): Healthy Controls - 5/15, 38.5 (28.0-37.0); Chronic Pancreatitis - 12/8, 49.5 (44.3-55.0); PDAC nonobstructed - 10/10, 67.5 (60.0-73.5); PDAC obstructed - 9/11, 68.0 (61.8-72.3); Benign Biliary Obstructed - 5/15, 65.5 (56.8-75.3). UKCTOCS cohort details - Gender M/F, Median Age in years (IQR): 0-1yr pre-diagnosis cases - (0/27, 68.3 (60.4-70.9); 0-1yr matched controls - 0/27, 66.3 (58.5-69.6). Duplicate measurements gave an average CV of 6.4\% (rho=0.966) for ICAM-1 and $9.9 \%$ (rho=0.991) for TIMP-1. Data were log transformed in order to remove skewness prior to analysis using JMP software, version 9.0.2. Groups were compared using ANOVA and t- tests; $\mathrm{p}$ values are shown on graphs for Liverpool cohort (* $\left.<0.05 ;{ }^{* *}<0.01 ;{ }^{* * *}<0.001,{ }^{* * * *}<0.0001\right)$.

\section{References}

1. Costello E, Greenhalf W, Neoptolemos JP. New biomarkers and targets in pancreatic cancer and their application to treatment. Nat Rev Gastroenterol Hepatol. 2012;9(8):435-44.

2. Tonack S, Jenkinson C, Cox T, Elliott V, Jenkins RE, Kitteringham NR, et al. iTRAQ reveals candidate pancreatic cancer serum biomarkers: influence of obstructive jaundice on their performance. British journal of cancer. 2013;108:1846-53.

3. Brand RE, Nolen BM, Zeh HJ, Allen PJ, Eloubeidi MA, Goldberg M, et al. Serum biomarker panels for the detection of pancreatic cancer. Clin Cancer Res. 2011;17:805-16.

4. Faca VM, Song KS, Wang H, Zhang Q, Krasnoselsky AL, Newcomb LF, et al. A mouse to human search for plasma proteome changes associated with pancreatic tumor development. PLoS Med. 2008;5:e123.

5. Pan S, Chen R, Crispin DA, May D, Stevens T, Mclntosh MW, et al. Protein alterations associated with pancreatic cancer and chronic pancreatitis found in human plasma using global quantitative proteomics profiling. Journal of proteome research. 2011;10:2359-76.

6. Yu KH, Barry CG, Austin D, Busch CM, Sangar V, Rustgi AK, et al. Stable isotope dilution multidimensional liquid chromatography-tandem mass spectrometry for pancreatic cancer serum biomarker discovery. Journal of proteome research. 2009;8:1565-76.

7. Pan S, Chen R, Brand RE, Hawley S, Tamura Y, Gafken PR, et al. Multiplex targeted proteomic assay for biomarker detection in plasma: a pancreatic cancer biomarker case study. J Proteome Res. 2012;11:1937-48. 
8. Mroczko B, Lukaszewicz-Zajac M, Wereszczynska-Siemiatkowska U, Groblewska M, Gryko M, Kedra B, et al. Clinical significance of the measurements of serum matrix metalloproteinase- 9 and its inhibitor (tissue inhibitor of metalloproteinase-1) in patients with pancreatic cancer: metalloproteinase- 9 as an independent prognostic factor. Pancreas. 2009;38:613-8.

9. Poruk KE, Firpo MA, Scaife CL, Adler DG, Emerson LL, Boucher KM, et al. Serum osteopontin and tissue inhibitor of metalloproteinase 1 as diagnostic and prognostic biomarkers for pancreatic adenocarcinoma. Pancreas. 2013;42:193-7.

10. Slater EP, Fendrich V, Strauch K, Rospleszcz S, Ramaswamy A, Matthai E, et al. LCN2 and TIMP1 as Potential Serum Markers for the Early Detection of Familial Pancreatic Cancer. Transl Oncol. 2013;6:99-103.

11. Menon U, Gentry-Maharaj A, Hallett R, Ryan A, Burnell M, Sharma A, et al. Sensitivity and specificity of multimodal and ultrasound screening for ovarian cancer, and stage distribution of detected cancers: results of the prevalence screen of the UK Collaborative Trial of Ovarian Cancer Screening (UKCTOCS). Lancet Oncol. 2009;10:32740 .

Conflicts of Interest: IJ and UM have a financial interest through UCL Business and Abcodia Ltd in the third party exploitation of trials biobanks, developed through their research at UCL. IJ has a consultancy arrangement with Becton Dickinson in the field of tumour markers and ovarian cancer. None of the other authors have any conflict of interest or other relationships or activities that could appear to have influenced the submitted work. 

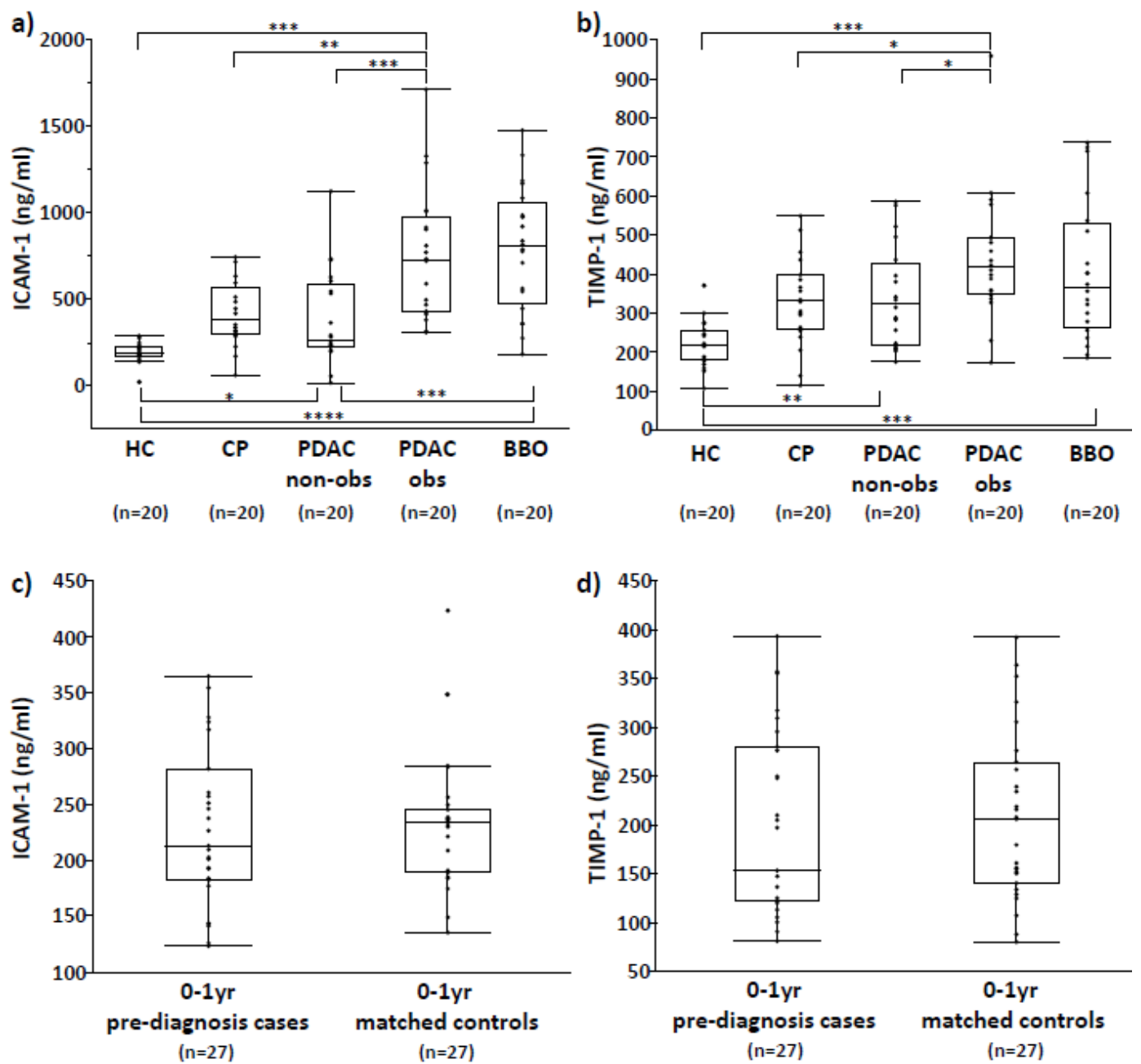